\title{
Impact of intraoperative stimulation mapping on high-grade glioma surgery outcome: a meta-analysis
}

\author{
Jasper Kees Wim Gerritsen ${ }^{1}$ (D) $\cdot$ Lidia Arends $^{2} \cdot$ Markus Klimek $^{3} \cdot$ Clemens Maria Franciscus Dirven $^{1}$ • \\ Arnaud Jean-Pierre Edouard Vincent ${ }^{1}$
}

Received: 18 September 2018 / Accepted: 7 November 2018 / Published online: 21 November 2018

(C) The Author(s) 2018

\begin{abstract}
Background Intraoperative stimulation mapping (ISM) using electrocortical mapping (awake craniotomy, AC) or evoked potentials has become a solid option for the resection of supratentorial low-grade gliomas in eloquent areas, but not as much for high-grade gliomas. This meta-analysis aims to determine whether the surgeon, when using ISM and AC, is able to achieve improved overall survival and decreased neurological morbidity in patients with high-grade glioma as compared to resection under general anesthesia (GA).

Methods A systematic search was performed to identify relevant studies. Adult patients were included who had undergone craniotomy for high-grade glioma (WHO grade III or IV) using ISM (among which AC) or GA. Primary outcomes were rate of postoperative complications, overall postoperative survival, and percentage of gross total resections (GTR). Secondary outcomes were extent of resection and percentage of eloquent areas.

Results Review of 2049 articles led to the inclusion of 53 studies in the analysis, including 9102 patients. The overall postoperative median survival in the AC group was significantly longer $(16.87$ versus 12.04 months; $p<0.001)$ and the postoperative complication rate was significantly lower $(0.13$ versus $0.21 ; p<0.001)$. Mean percentage of GTR was significantly higher in the ISM group (79.1\% versus $47.7 \%, p<0.0001)$. Extent of resection and preoperative patient KPS were indicated as prognostic factors, whereas patient KPS and involvement of eloquent areas were identified as predictive factors.

Conclusions These findings suggest that surgeons using ISM and AC during their resections of high-grade glioma in eloquent areas experienced better surgical outcomes: a significantly longer overall postoperative survival, a lower rate of postoperative complications, and a higher percentage of GTR.
\end{abstract}

Keywords Awake craniotomy $\cdot$ Glioblastoma $\cdot$ Extent of resection $\cdot$ Morbidity $\cdot$ Mortality

This article is part of the Topical Collection on Brain Tumors

Electronic supplementary material The online version of this article (https://doi.org/10.1007/s00701-018-3732-4) contains supplementary material, which is available to authorized users.

Jasper Kees Wim Gerritsen

j.gerritsen@erasmusmc.nl

1 Department of Neurosurgery, Erasmus Medical Center Rotterdam, 's-Gravendijkwal 230, 3015 CE Rotterdam, The Netherlands

2 Department of Biostatistics, Erasmus Medical Center Rotterdam, Rotterdam, The Netherlands

3 Department of Anesthesiology, Erasmus Medical Center Rotterdam, Rotterdam, The Netherlands

\section{Introduction}

Glioblastomas (WHO IV glioma) are devastating tumors with one of the worst prognoses in oncology. The median survival after surgery and combined treatment with chemo- and radiotherapy ranges from 12 to 15 months and no curative therapy is currently available [8, 23]. Multiple studies show that extent of resection of the contrast enhancing part of the tumor improves survival in patients with GBM [15-18, 20-22, 24]. Further analyses showed that patients who previously had complete resections derived the most benefit from the temozolomide (TMZ) regimen compared with those who had had incomplete resection [1]. Thus, in addition to the survival benefit associated with maximum cytoreductive surgery, 
such surgery seems essential for the efficacy of modern adjuvant treatment. More than $50 \%$ of GBMs are located near or in eloquent areas of the brain. Damaging these areas during surgery can lead to severe and permanent neurological deficits that seriously impact the quality of life. Therefore, when resecting GBMs in these areas, they are usually not operated as aggressive as possible, due to the chance of seriously damaging the patient with a rather low life expectancy [13, 15-18, 20, 23]. However, patients with partial or subtotal resections will benefit less from radio- and chemotherapy as compared to patients with total resections [15-18, 20-22, 24].Intraoperative stimulation mapping (ISM) allows the surgeon to prevent damage to eloquent cortical and subcortical areas during resection $[2,19]$. There is compelling evidence that surgeons using ISM experience increased resection percentage while preserving quality of life in low-grade glioma (LGG). We expect that the use of awake craniotomy by surgeons therefore is also of important value in the surgery of GBM, and in a similar fashion can optimize the extent of resection and preserve quality of life, thereby improving survival in these patients $[2,5-7,9-12,14$, 19].

The usefullness of ISM by surgeons and its impact on neurologic outcome has been evaluated mainly for mostly lowgrade gliomas or as a descriptive review. In this article, a metaanalysis is performed to compare the surgeon's use of intraoperative stimulation mapping (among which awake craniotomy, AC) versus general anesthesia for the resection of highgrade glioma.

\section{Methods}

\section{Search strategy}

A computer-aided search of Embase, Medline (OvidSP), Web of Science, the Cochrane Library, Pubmed, and Google Scholar was performed to identify relevant studies. The search terms used were (craniotomy OR surgery OR surgical approach OR surgical patient OR surgical technique OR brain surgery OR brain tumor OR cancer surgery OR neurosurgery OR intraoperative period) AND (wakefulness OR sedation OR conscious sedation OR consciousness OR arousal OR local anesthesia OR local anesthetic agent OR electrostimulation OR sensorimotor function OR (stimulation AND brain cortex)) AND (glioma OR brain tumor OR brain cancer OR intracranial tumor OR gliobastoma OR ((brain OR intracranial OR supratentorial OR cortex OR cortical) NEAR (tumor OR tumour OR cancer OR lesion)) NOT (conference abstract OR letter OR note OR editorial) AND (english). The publication period was restricted to January 1, 1990, to April 1,2017 . One reviewer (JKWG) performed the initial search in association with a biomedical information specialist of the library service of Erasmus Medical Centre, who verified the search. Reference lists of the studies were searched for additional valuable studies.

\section{Study selection criteria}

To be included in the meta-analysis, all studies had to have examined the effects of resective glioma surgery with or without the use of ISM by surgeons. Studies were reviewed that used resective glioma surgery to improve the prognosis in patients with high-grade glioma (WHO III-IV). Only complemented studies meeting the PICO format of this study where full-text versions were available were included. PICO (Population Intervention Comparison Outcome) restrictions were made for population (patients under 18 years old were excluded), intervention (fMRI, DTI, MSI, neuronavigation, or ultrasound were not considered ISM), and outcome: eligible primary outcomes were survival, extent of resection (percentage gross total resection-GTR), and complication rate. Studies were exluded if they included patients with glioma grading other than $\mathrm{WHO}$ grade 3 or 4; patients under 18 years old; when the pathohistology of the tumors was not specified; when the article was of review-, editorial-, commentary-, short report-format or was a chapter in a book; and when no abstract was available (Fig. 1, flowchart).

\section{Outcome measures and definitions}

The primary outcome measures were the event rate of postoperative complications, overall survival, and percentage GTR. Postoperative complications were noted as such as defined by colleagues de Witt Hamer et al. [7] Complications were not categorized according to severity and timing of assessment. Complications were eligible as such when they emerged postoperatively; when pre-operative symptoms worsened; or when they were part of a worsening of the patients' condition postoperatively. The percentage of patients in whom GTR was obtained according to postoperative neuroimaging was also extracted. Furthermore, data regarding patient KPS and the percentage of craniotomies concerning eloquent areas was collected. Sources related to publication, population, or management characteristics were distinguished. Publication-related characteristics were publication year, continent, and study setting. Patient population-related characteristics were mean age and percentage of eloquently located gliomas. Treatmentrelated characteristics were intraoperative techniques and sort of anesthesia (awake or general anesthesia). Intraoperative techniques included percentage of patients with resections using ISM. For ISM, electrocortical mapping was distinguished from motor- or somatosensory evoked potentials (MEP, SEP). 


\section{Statistical analysis}

Differences between the ISM group (with or without AC) and GA group for the primary outcomes were tested: (1) overall postoperative survival and (2) rate of postoperative complications. Analysis of the the data set for primary outcomes was based on non-parametrics tests; for number of complications, the Mann-Whitney test was used, whereas for survival, the log-rank test was used and for the difference in percentage GTR between groups, a two-tailed $t$ test. No adjustment for multiple testing has been done. The significance level was set to $5 \%$. Forest plots were made with SPSS (Version 24.0; IBM Analytics). Analysis of the relationships between factors was done using a mixed effects regression analysis (unrestricted ML).

\section{Results}

\section{Study characteristics}

The search strategy yielded 3785 publications, of which 2026 remained after duplicates were removed. Twenty-three additional recoreds were identified through alternative search strategies, mainly by searching the reference lists of the studies, increasing the total number of records identified to 2049 .
Following screening by title and abstract, 146 articles were considered relevant and were assessed for eligibility. Review of these 146 articles led to the inclusion of 53 studies in the analysis, including 9102 patients. A total of 1260 patients were operated using ISM; 7842 patients were operated under general anesthesia. The study characteristics of the 53 publications are listed in the Data Supplement. Not all studies allowed extraction of all end points. The complications mainly consisted of motor and language deficits (Data Supplement). The cohorts varied between 9 and 1229 patients. Included articles were publicated between 1999 and 2016. Twenty-two articles were of European origin, 21 articles were of North-American origin, eight articles were of Asian origin, two articles were of South-American origin, and one article was of Middle-East origin. Fortyeight studies were performed in a university setting $(91 \%)$. Eleven studies used electrostimulation (sub) cortical mapping intraoperatively. Eleven studies used evoked potentials (such as MEPs, SEPs) intraoperatively. Four studies used awake craniotomy as anesthetic modality. The mean age of the study populations differed between 49.0 and 78.0 years. Percentage of eloquently located gliomas differed between 0 and $100 \%$. The percentage of patients in whom GTR was obtained differed between 6 and $96 \%$. The overall postoperative median survival of patients following diagnosis differed between 4.5 and
Fig. 1 Flowchart of total records identified through database searching

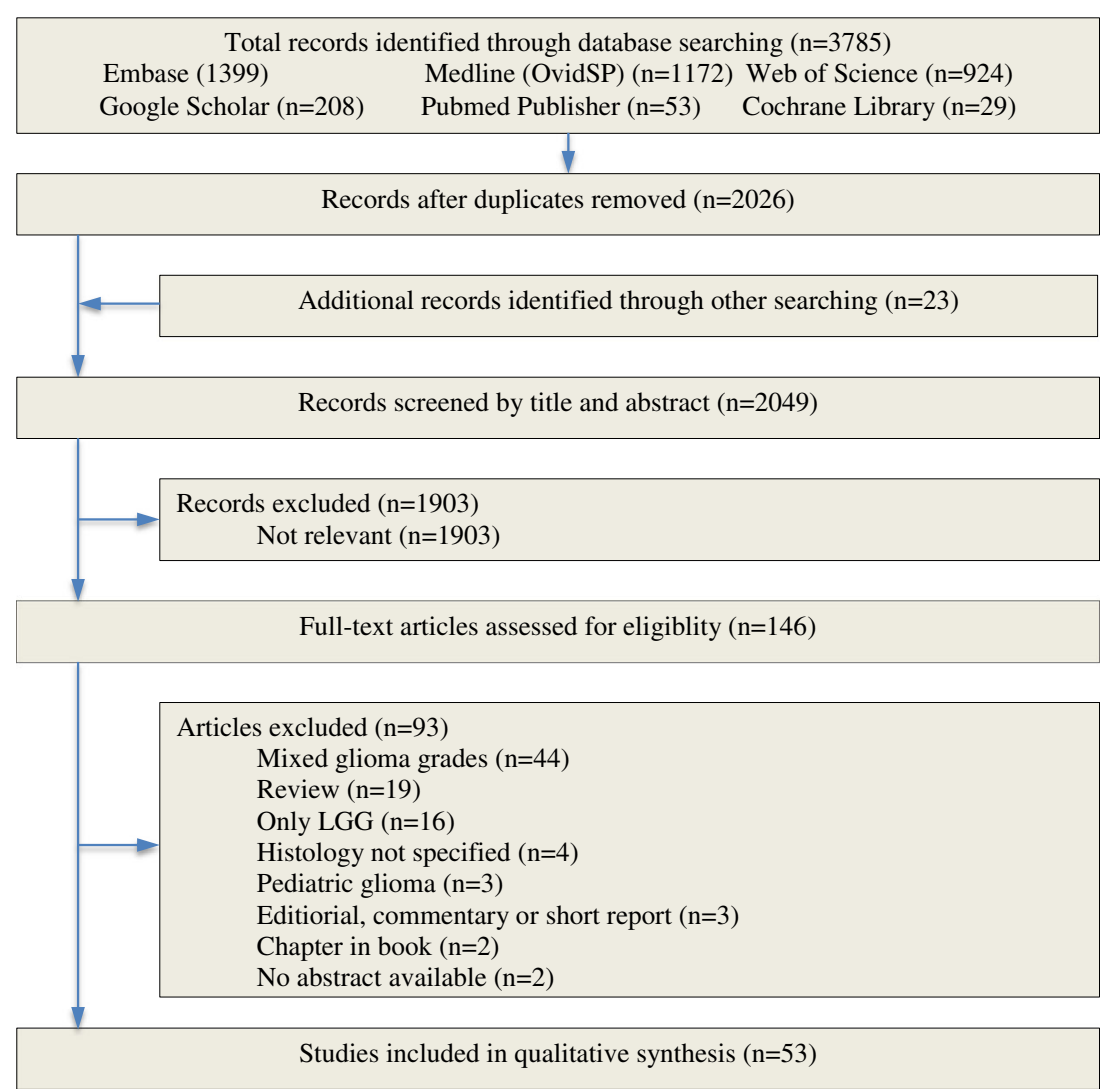


16.3 months. Postoperative complication rates differed between 0.0 and 0.64 .

\section{Overall survival}

The median overall survival rates for each study are provided in Table 1 of the Data Supplement. Studies evaluating craniotomy under GA with data on overall survival $(n=17)$ included 4390 patients with a median overall survival of 12.04 months ( $\mathrm{SE}=1.14 ; 95 \%$ CI 9.80-14.28). Studies evaluating craniotomy with ISM with available data $(n=5)$ included 279 patients with a median overall survival of 15.53 months ( $\mathrm{SE}=1.68$; 95\% CI 12.2418.82). Studies evaluating awake craniotomy (subgroep of ISM) with available data $(n=3)$ included 210 patients with a median overall survival of 16.87 months ( $\mathrm{SE}=$ $0.75 ; 95 \%$ CI 15.40-18.34). The median survival in the ISM group was almost 3.5 months longer than in the GA group, but this was not significant $(p=0.085)$. The median survival in the awake group was more than 4.5 months longer than in the GA group, which was significant $(p<0.001)$. Forest plots for median overall survival rates are displayed in Fig. 2.

\section{Extent of resection and survival}

Eighteen studies evaluated the extent of resection (expressed as percentage of craniotomies in which gross total resection (GTR) was achieved) in correlation with overall survival. Percentages of craniotomies in which GTR was achieved in each study are provided in Table 1 of the Data Supplement. Using a mixed effects regression with unrestricted ML, a significant positive relation was found between extent of resection and overall survival $(b=0.11 ; \mathrm{SE}=0.04 ; p=0.012)$ (Fig. 3), indicating extent of resection as a major prognostic factor in high-grade glioma surgery.

\section{Preoperative patient KPS and survival}

Forty-five studies evaluated preoperative patient KPS (Karnofsky Performance Score) in correlation with overall survival. Preoperative patient KPS are provided in Table 1 of the Data Supplement. Using a mixed effects regression with unrestricted ML, a significant positive relation was found between preoperative patient KPS and overall survival $(b=0.61$; $\mathrm{SE}=0.13 ; p<0.001)$, indicating preoperative patient KPS as a major prognostic factor in high-grade glioma surgery.

\section{Postoperative complications}

The postoperative complication rates for each study are provided in Table 1 of the Data Supplement. Studies evaluating craniotomy under GA with data on postoperative complications $(n=19)$ included 5826 patients with a total of 1250 postoperative complications. In this group, the postoperative complication rate was 0.21 (95\% CI 0.20-0.23). Studies evaluating craniotomy with ISM with available data $(n=9)$ included 430 patients with a total of 54 postoperative complications. In this group, the postoperative complication rate was 0.13 (95\% CI $0.10-0.16)$. The complication rate in the ISM group was significantly lower than in the GA group $(p<0.001)$.

\section{Extent of resection}

The extent of resection is expressed as percentage of GTR obtained for each study. The data are provided in Table 1 of the Data Supplement. Studies evaluating craniotomy under GA with data on extent of resection $(n=24)$ included 6880 patients. In 3283 cases, GTR was obtained. In this group, the mean percentage of GTR was 47.7\% (95\% CI 40.4-55.5). Studies evaluating craniotomy with ISM with available data ( $n=6$ ) included 369 patients. In 292 cases, GTR was obtained. In this group, the mean percentage of GTR was $79.1 \%$ (95\% CI 69.8-88.4). The mean percentage of GTR in the ISM group was significantly higher than in the GA group $(p<0.001)$.

\section{Extent of resection and postoperative complications}

Fifteen studies evaluated extent of resection (expressed as percentage of craniotomies in which gross total resection (GTR) was achieved) in correlation with postoperative complications. Percentages of craniotomies in which GTR was achieved in each study are provided in Table 1 of the Data Supplement. Using a mixed effects regression with unrestricted ML, no relation was found between extent of resection and overall survival ( $b=-0.018$; SE-0.012; $p=0.132$ ) (Fig. 4), indicating that achieving a higher extent of resection does not yield a higher rate of postoperative complications.

\section{Patient KPS and postoperative complications}

Ten studies evaluated preoperative patient KPS in correlation with postoperative complications. Percentages of eloquent areas in each study are provided in Table 1 of the Data Supplement. Using a mixed effects regression with unrestricted ML, a significant positive relation was found between patient KPS and postoperative complications $(b=-0.095 ; \mathrm{SE}=0.039 ; p=0.014)$, indicating preoperative patient KPS as a major predictive factor for postoperative complications in high-grade glioma surgery. 


\section{GA group}

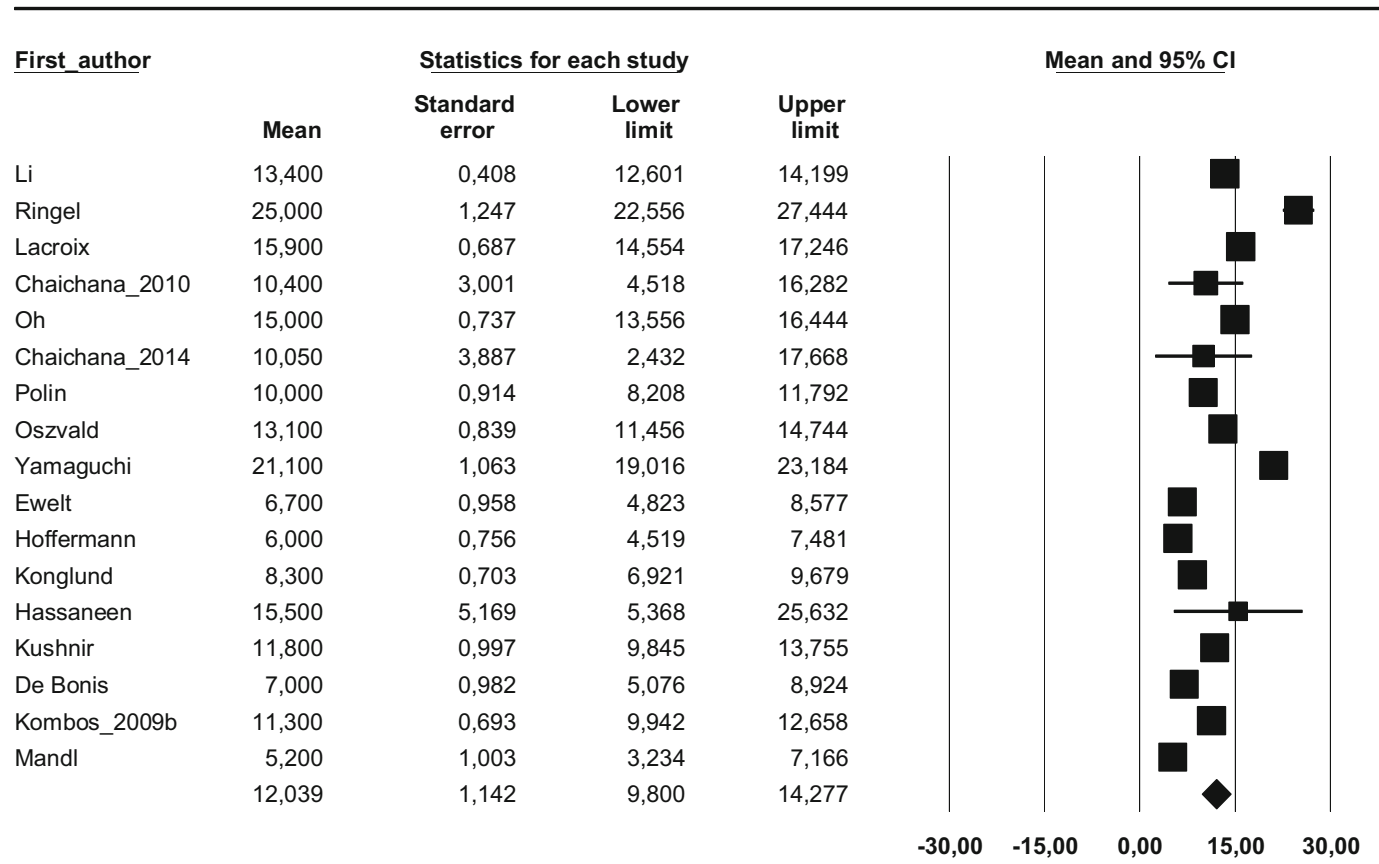

ISM group

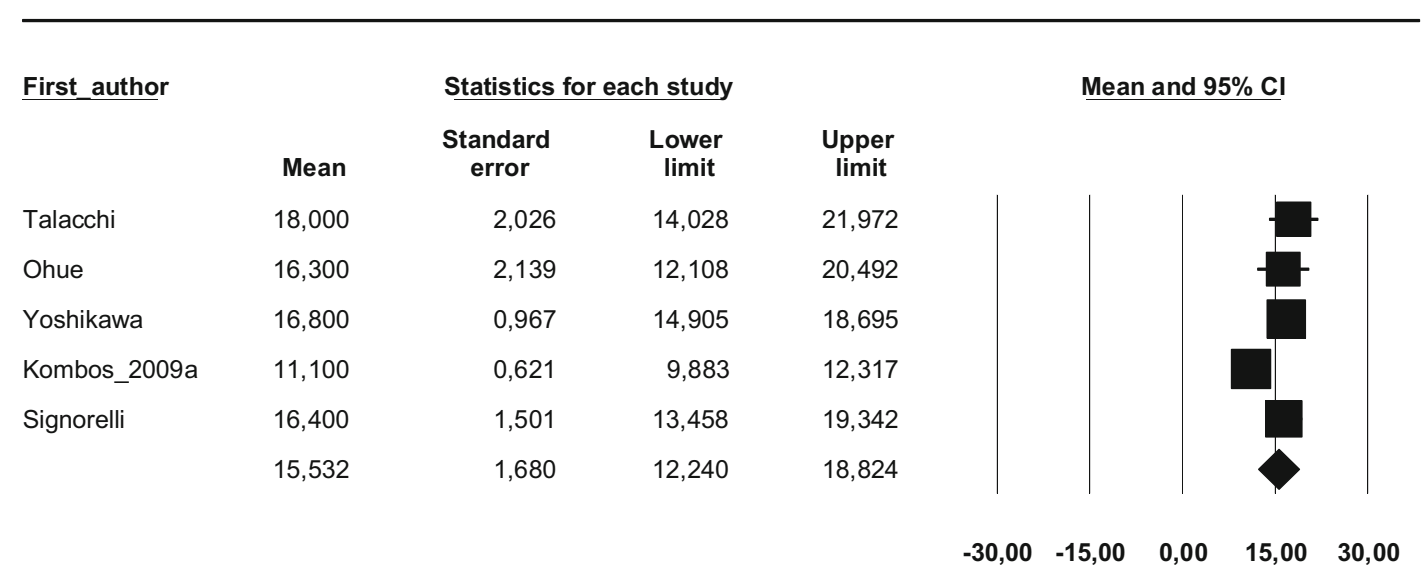

Awake group

First_author

$\begin{array}{lr} & \text { Mean } \\ \text { Talacchi } & 18,000 \\ \text { Yoshikawa } & 16,800 \\ \text { Signorelli } & 16,400 \\ & 16,865\end{array}$

Statistics for each study

$\begin{array}{rr}\begin{array}{c}\text { Standard } \\ \text { error }\end{array} & \begin{array}{c}\text { Lower } \\ \text { limit }\end{array} \\ 2,026 & 14,028 \\ 0,967 & 14,905 \\ 1,501 & 13,458 \\ 0,754 & 15,387\end{array}$

Mean and $95 \% \mathrm{Cl}$

Upper

limit

21,972

18,695

19,342

18,344

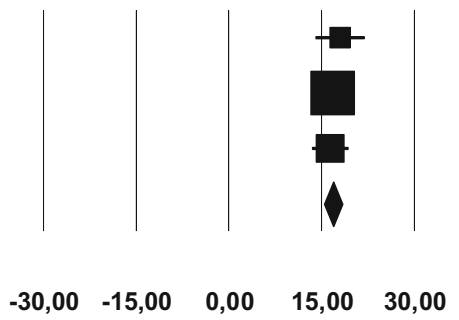

Fig. 2 Forest plot 
Fig. 3 Extent of resection and overall survival

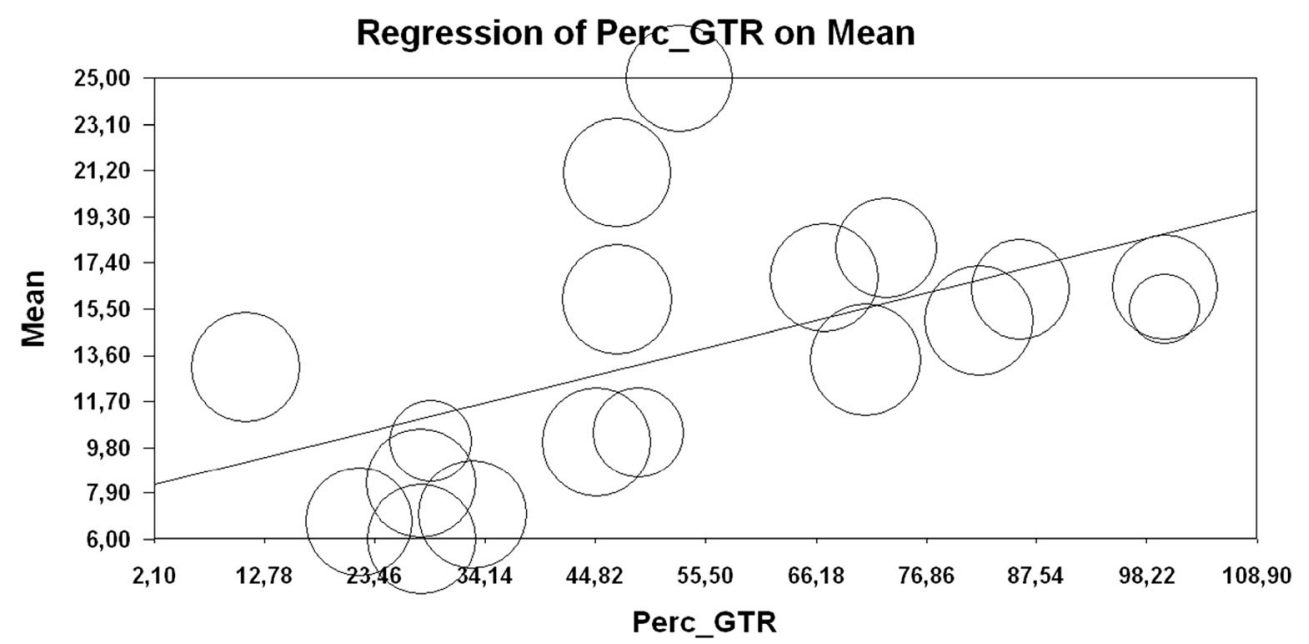

\section{Eloquent areas and postoperative complications}

Eleven studies evaluated the percentage of eloquent areas in correlation with postoperative complications. Percentages of eloquent areas in each study are provided in Table 1 of the Data Supplement. Using a mixed effects regression with unrestricted ML, no overall relation was found between the percentage of eloquent areas and postoperative complications $(b=-0.009$; $\mathrm{SE}=0.013 ; p=0.475$ ). However, a significant relation was found when evaluating only studies investigating craniotomies under GA, indicating that a higher percentage of eloquent areas was significantly positively related with a higher postoperative complication rate $(b=0.044 ; \mathrm{SE}=0.007 ; p<0.001)$.

\section{Discussion}

This meta-analysis shows that patients who had been operated by surgeons using AC as ISM for a single supratentorial highgrade glioma had a significant longer overall postoperative median survival (more than 4.5 months longer) and were subject of less postoperative complications in eloquent areas ( 0.13 versus 0.21$)$. Furthermore, the percentage of resections in which GTR was obtained was significantly higher in the ISM group as compared to the GA group ( $47.7 \%$ versus 79.1 , $p<0.001)$. The use of ISM and AC by surgeons is safe, as a greater extent of resection did not yield a higher rate of complications. Moreover, extent of resection and preoperative patient KPS were indicated as prognostic factors, whereas patient KPS and the involvement of eloquent areas were identified as predictive factors. These results suggest that the use of ISM (AC in particular) by surgeons should be implemented as a routine operation for surgery of high-grade tumors near eloquent areas of the brain.

It is important to recognize the ifs and buts of the use of adjunct surgical techniques such as ISM and AC. The results yielded by the use of such techniques are only as good as the surgeon who uses these techniques. The fact that no technique can ever replace knowledge, experience and skill should be acknowledged, valued, and acted upon accordingly. Mapping and monitoring during glioma resections are useless if the surgeon is not familiar with using these techniques.
Fig. 4 Extent of resection and postoperative complication rate

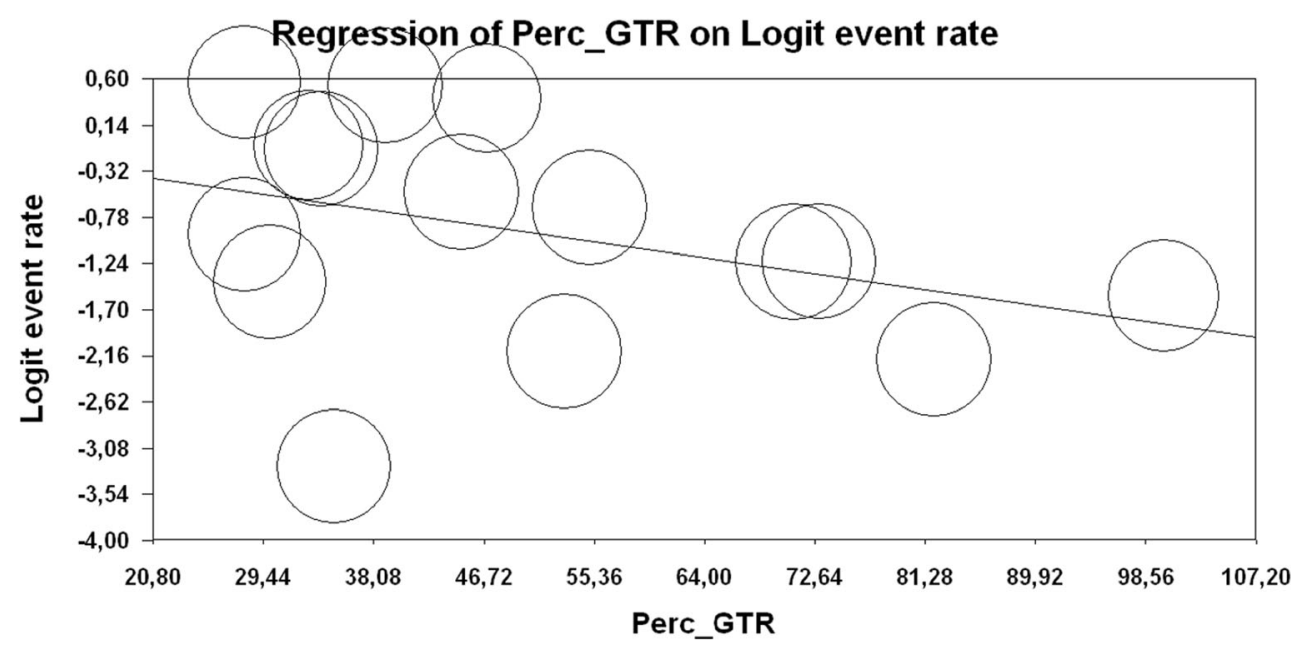


Implementing new techniques takes practice and will inevitably come with a certain learning curve regarding both technical use and case selection.

Neurosurgeons have a daunting task: resecting the tumor with an extent as great as possible, while simultaneously minimizing the risk for postoperative complications and especially neurological morbidity. Surgeons use ISM to maximize resection, primarily to increase the patient's survival while minimizing the chances of morbidity and loss of neurological function [2,24]. AC is the most frequently used form of ISM, by using electrocortical and subcortical mapping to differ eloquent brain tissue from brain or tumor tissue that is safe to resect. Hereby, surgeons try to maximize the extent of resection with at the same time minimizing the risk of postoperative complications. To date, surgeons use ISM and AC in particular for the resection of low-grade gliomas because of the usually near-eloquent location of these tumors [4, 13]. Only few studies have evaluated the use of these techniques in highgrade gliomas, as is reflected in the studies included (see also Table 1, Data Supplement). We showed that surgeons using AC can significantly contribute to this goal by preserving the quality of life of these patients and decreasing the risk of postoperative morbidity when operating in eloquent areas, while increasing extent of resection and maximizing postoperative survival.

To the best of our knowledge, this is the first study that systematically investigates the use of ISM and AC by surgeons in high-grade glioma surgery only.

A study and meta-analysis very similar to ours, conducted by De Witt Hamer et al., included 8091 patients with supratentorial infiltrative glioma (high- and lowgrade glioma) that were resected by surgeons using ISM or not [7]. They found that glioma resections in which the surgeon had used ISM were associated with fewer late major neurologic deficits. These findings are in accordance with our results evaluating high-grade glioma resections, since we found that surgeons using $\mathrm{AC}$ as ISM experienced decreased rates of postoperative complications in eloquent areas.

Sacko et al. prospectively compared surgeons using AC versus craniotomy under GA for resection of supratentorial lesions including 575 glioma patients [19]. They found that patients with tumors in eloquent areas revealed a significantly better neurological outcome and extent of resection in the AC group than the GA group. Although this study also includes low-grade glioma patients, it is one of the largest prospective studies comparing surgeons using AC and craniotomy under GA head-to-head for postoperative outcomes in glioma surgery. We found similar results after our data analysis, suggesting a role for the use of $\mathrm{AC}$ by surgeons in resections for highgrade glioma - especially in eloquent areas - to improve outcomes after craniotomies.
Chaichana et al. conducted a retrospective study at the Johns Hopkins University to develop a prognostic grading system in glioblastoma patients [3]. They found that (among others) a poor preoperative performance status proved to be a strong prognostic factor in glioblastoma surgery. In accordance with this findings, we found that preoperative patient KPS was not only indicated as a prognostic factor, but also a predictive factor (a poor preoperative KPS indicating an increased risk of postoperative complications). These results underline the importance of identifying subgroups of patients within the high-grade glioma patient population and the role ISM/AC use by surgeons can play in optimalization of surgery outcomes.

This study should be interpreted whitin the limitations of a meta-analysis based on observational studies. The selected publications are observational or retrospective in nature and therefore subject to selection bias, publication bias, and subjective outcome assesments, as mentioned before by de Witt et al. [7]. We therefore advise a randomized controlled trial where awake craniotomy with ISM is compared to surgery under general anesthesia for GBM near eloquent areas. Primary outcomes should be focused on neurological morbidity and extent of resection.

Publication bias can result in our findings being rather precise, with small 95\% CIs, but less accurate as an estimation of the ground truth of outcome in general neurosurgical practice, since published data does not necessarily reflect general clinical practice. For example, the overall low rate of mortality is known to correlate with large-volume centers. We therefore think our results may apply to high-grade glioma patients treated in large-volume academic hospitals dedicated to neuro-oncology. Secondly, the selection bias of our findings can be expected due to patient selection with various indications for surgical intervention. However, we minimized the risk for this bias by our vast amount of data and number of included studies of patients.

\section{Conclusions}

Surgeons resecting high-grade glioma with ISM are able to achieve a higher percentage of GTR, and the use of AC by surgeons is associated with significantly longer overall postoperative survival with less postoperative complications as compared with craniotomy under GA. The greater extent of resection achieved by mapping techniques did not yield a higher rate of complications. Furthermore, extent of resection and preoperative patient KPS were indicated as prognostic factors, whereas patient KPS and the involvement of eloquent areas were identified as predictive factors. Our findings confirm preliminary findings of other authors with smaller group sizes and elaborate on large studies with both low-grade and high-grade patient cohorts. Future studies should focus on 
evaluating the role of the use of AC by surgeons in the treatment of high-grade glioma and optimize risk stratification using prognostic factors. Subgroups of patients should be identified that might benefit the most from extensive surgery and AC. If future studies confirm and elaborate on the results presented in this study, the role of awake craniotomies in neurooncology should be revisited and expanded.

Acknowledgments We would like to thank W. Bramer, biomedical information specialist at the Erasmus MC Library for assisting us with the computer-aided systematic search.

Author's contribution Study concept: JG, AV.

Study design: JG, LA, AV.

Data acquisition: JG.

Quality control of data and algorithms: JG, LA.

Data analysis and interpretation: JG, AV, LA.

Statistical analysis: LA.

Manuscript preparation: JG, LA, MK, CD, AV.

Manuscript editing: JG, MK, AV.

Manuscript review: MK, CD, AV.

Funding Financial nor material support have been received.

\section{Compliance with ethical standards}

Conflict of interest All authors certify that they have no affiliations with or involvement in any organization or entity with any financial interest (such as honoraria; educational grants; participation in speakers' bureaus; membership, employment, consultancies, stock ownership, or other equity interest; and expert testimony or patent-licensing arrangements), or non-financial interest (such as personal or professional relationships, affiliations, knowledge, or beliefs) in the subject matter or materials discussed in this manuscript. I confirm that there are no known conflicts of interest associated with this publication and there has been no significant financial support for this work that could have influenced its outcome.

Ethical approval For this type of study, formal consent is not required.

This article does not contain any studies with human participants performed by any of the authors.

No portions of the contents of this paper have been presented nor published previously.

Open Access This article is distributed under the terms of the Creative Commons Attribution 4.0 International License (http:// creativecommons.org/licenses/by/4.0/), which permits unrestricted use, distribution, and reproduction in any medium, provided you give appropriate credit to the original author(s) and the source, provide a link to the Creative Commons license, and indicate if changes were made.

\section{References}

1. Black PM, Ronnen SF (1987) Cortical mapping for defining the limits of tumor resection. Neurosurgery 20:914-919

2. Brown TJ, Brandmeir NJ, Church EW, Brennan MC, Li M, Rakszawski KL et al (2016) Association of the extent of resection with survival in glioblastoma: a systematic review and meta-analysis. JAMA Oncol 2:1460-1469

3. Chaichana K, Olivi A, Parker S, Quiñones-Hinojosa A (2010) A proposed classification system that projects outcomes based on preoperative variables for adult patients with glioblastoma multiforme. J Neurosurg 112:997-1004

4. Chang EF, Barbaro NM, Berger MS, Chang SM, Clark A, McDermott MW et al (2011) Functional mapping-guided resection of low-grade gliomas in eloquent areas of the brain: improvement of long-term survival. Clinical article. J Neurosurg 114:566-573

5. Danks RA, Aglio LS, Gugino LD, Black PM (2000) Craniotomy under local anesthesia and monitored conscious sedation for the resection of tumors involving eloquent cortex. J Neuro-Oncol 49: 131-139

6. De Benedictis A, Moritz-Gasser S, Duffau H (2010) Awake mapping optimizes the extent of resection for low-grade gliomas in eloquent areas. Neurosurgery 66:1074-1084 discussion 1084

7. De Witt Hamer PC, Berger MS, Duffau H, Robles SG, Zwinderman AH (2012) Impact of intraoperative stimulation brain mapping on glioma surgery outcome: a meta-analysis. J Clin Oncol 30:2559-2565

8. DeAngelis LM (2001) Brain tumors. N Engl J Med 344:114-123

9. Duffau H (2009) A personal consecutive series of surgically treated 51 cases of insular WHO grade II glioma: advances and limitations. J Neurosurg 110:696-708

10. Duffau H, Capelle L, Denvil D, Sichez N, Gatignol P, Lopes M, Mitchell MC, Sichez JP, Van Effenterre R (2003) Functional recovery after surgical resection of low grade gliomas in eloquent brain: hypothesis of brain compensation. J Neurol Neurosurg Psychiatry 74:901-907

11. Duffau H, Capelle L, Denvil D, Sichez N, Gatignol P, Taillandier L, Lopes M, Mitchell MC, Roche S, Muller JC, Bitar A, Sichez JP, van Effenterre R (2003) Usefulness of intraoperative electrical subcortical mapping during surgery for low-grade gliomas located within eloquent brain regions: functional results in a consecutive series of 103 patients. J Neurosurg 98:764-778

12. Duffau H, Lopes M, Arthuis F, Bitar A, Sichez JP, Van Effenterre R, Capelle L (2005) Contribution of intraoperative electrical stimulations in surgery of low grade gliomas: a comparative study between two series without (1985-96) and with (1996-2003) functional mapping in the same institution. J Neurol Neurosurg Psychiatry 76:845-851

13. Gupta DK, Chandra PS, Mahapatra AK, Mehta VS, Ojha BK (2007) Sharma BS (2007) awake craniotomy versus surgery under general anesthesia for resection of intrinsic lesions of eloquent cortex - a prospective randomized study. Clin Neurol Neurosurg 109: 335-343

14. Hervey-Jumper SL, Li J, Lau D, Molinaro AM, Perry DW, Meng L, Berger MS (2015) Awake craniotomy to maximize glioma resection: methods and technical nuances over a 27 -year period. J Neurosurg 123:325-339

15. Jeremic B, Grujicic D, Antunovic V, Djuric L, Stojanovic M, Shibamoto Y (1994) Influence of extent of surgery and tumor location on treatment outcome of patients with glioblastoma multiforme treated with combined modality approach. J NeuroOncol 21:177-185

16. Keles GE, Anderson B, Berger MS (1999) The effect of extent of resection on time to tumor progression and survival in patients with glioblastoma multiforme of the cerebral hemisphere. Surg Neurol 52:371-379

17. Keles GE, Lamborn KR, Chang SM, Prados MD, Berger MS (2004) Volume of residual disease as a predictor of outcome in adult patients with recurrent supratentorial glioblastomas multiforme who are undergoing chemotherapy. J Neurosurg 100:41-46 
18. Lacroix M, Abi-Said S, DeMonte F, Fourney DR, Gokaslan ZL, Shi W et al (2001) A multivariate analysis of 416 patients with glioblastoma multiforme: prognosis, extent of resection, and survival. J Neurosurg 95:190-198

19. Sacko O, Brauge D, Brenner A, Lauwers-Cances V, Roux FE, Sesay M (2011) Awake craniotomy vs surgery under general anesthesia for resection of supratentorial lesions. Neurosurgery 68: 1992-1998

20. Sanai N, Berger MS (2008) Glioma extent of resection and its impact on patient outcome. Neurosurgery 62:753-764

21. Smith JS, Cha S, Chang EF, Chang SM, Lamborn KR, Prados MD et al (2008) Role of extent of resection in the long-term outcome of low-grade hemispheric gliomas. J Clin Oncol 26:1338-1345

22. Stummer W, Meinel T, Pichlmeier U, Reulen HJ, Schumacher W, Tonn JC et al (2008) Extent of resection and survival in glioblastoma multiforme: identification of and adjustment for bias. Neurosurgery 62:564-576

23. Stupp R, Mason WP, van den Bent MJ, Weller M, Fisher B, Taphoorn MJ, Belanger K, Brandes AA, Marosi C, Bogdahn U, Curschmann J, Janzer RC, Ludwin SK, Gorlia T, Allgeier A, Lacombe D, Cairncross JG, Eisenhauer E, Mirimanoff RO (2005) Radiotherapy plus concomitant and adjuvant temozolomide for glioblastoma. N Engl J Med 352:987-996

24. Vourinen V, Hinkka S, Jääskeläinen J, Farkkila M (2003) Debulking or biopsy of malignant glioma in elderly people - randomized study. Acta Neurochir 145:5-10

\section{Importance of the study}

Aggressive surgery is warranted in glioblastoma surgery, since the extent of resection improves survival in this patient group. However, since the majority of glioblastomas are located in eloquent areas, operating aggressively is often impossible due to imminent negative consequences for survival and quality of life (QoL). Hence, a surgical technique optimizing resection percentage of these tumors while preventing neurological deficits is necessary. Implementation of the use of intraoperative stimulation mapping (ISM) and awake craniotomy (AC) by surgeons made this endeavor possible in eloquently located low-grade glioma. Therefore, these techniques may be beneficial for surgeons resecting glioblastoma in eloquent areas as well. The current literature regarding the use of these techniques in glioblastoma surgery is insufficient. Our study advances current research by summarizing the evidence for the use of these techniques in glioblastoma surgery by investigating more than 9000 patients, hereby laying the foundation for further research regarding the evidence-based use of these techniques in glioblastoma surgery. 